\title{
El viaje como recurso educativo. Estudio de caso de la Expedición Tahina-Can
}

\author{
Travel as an educational resource. \\ Case study of the Tahina-Can Expedition
}

\author{
Santiago Tejedor \\ Universidad Autónoma de Barcelona \\ santiago.tejedor@uab.cat \\ Código Orcid: http://orcid.org/0000-0002-5539-9800 \\ Santiago Giraldo Luque \\ Universidad Autónoma de Barcelona \\ santiago.giraldo@uab.cat \\ Código Orcid: https://orcid.org/0000-0003-0024-7081
}

\begin{abstract}
Resumen
El trabajo analiza las posibilidades del viaje como recurso y método educativo innovador a partir del estudio de caso de la Expedición Tahina-Can, un proyecto de periodismo de viajes impulsado por la Universidad Autónoma de Barcelona (UAB) desde el año 2004 y que ha permitido a cerca de 700 estudiantes participar en una expedición académica que aglutina talleres de producción de contenidos en el ámbito de la redacción digital, la televisión, la fotografía y la radio. La iniciativa ha obtenido el primer premio por la Fundación Telefónica en la categoría de "Aprendizaje en la Red". El estudio propone la conceptualización del viaje como práctica constructivista que asume los retos del perfil del estudiante del siglo XXI. A través de la metodología del estudio de caso, describe las particularidades del proyecto de viajes al tiempo que analiza los resultados y el impacto del mismo a partir de grupos focales desarrollados con la participación de los expedicionarios que han hecho parte de los viajes de la Expedición. El estudio concluye, entre otros aspectos, que el viaje es considerado como una práctica de aprendizaje activo y que constituye un estímulo para los estudiantes. La experiencia potencia el compromiso y a la entrega de los participantes con su propio aprendizaje y permite el desarrollo avanzado de diferentes roles y competencias periodísticas profesionales.
\end{abstract}

\section{Palabras clave}

Periodismo, educación, viaje, comunicación, internet, aprendizaje.

Forma sugerida de citar: Tejedor, Santiago, y Giraldo Luque, Santiago (2019). El viaje como recurso educativo. Estudio de caso de la Expedición Tahina-Can. Universitas, 30, pp. 19-39. 


\begin{abstract}
The work analyses the possibilities of travel as a resource and innovative educational method based on the case study of the Tahina-Can Expedition, a travel journalism project promoted by the Autonomous University of Barcelona (UAB). Since 2004, it has allowed about 700 students to participate in an academic expedition that brings together content production workshops in the field of digital writing, television, photography and radio. The initiative has obtained the first prize by the Telefónica Foundation in the category of "Learning on the Net". The study proposes the conceptualization of the trip as a constructivist practice that assumes the challenges of the 21st century student profile. Through the methodology of the case study, the article describes the particularities of the travel project, as well as it analyses its results and its impact. This is done through the technique of focus groups, which are developed with the participation of the expedition members who have taken part in the trips of Tahina-Can. The study concludes, among other aspects, that the trip is considered as an active learning practice and that constitutes a stimulus for the students. The experience enhances the commitment of the participants with their own learning process and it allows the advanced development of different professional journalistic roles and competences.
\end{abstract}

Keywords

Journalism, education, travel, communication, internet, learning.

\title{
Comunicación, educación y viaje
}

La universidad y, por ende, la educación necesita ser objeto de una somera y profunda reflexión. El profesor e investigador de Políticas Públicas de la Universidad de Harvard, Dan Levy (Amiguet, 2017), el director del eLearning Center de la UOC, Lluis Pastor (Farreras, 2016), el experto en innovación y miembro de Singularity University David Roberts (Torres, 2016) o el decano de la Universidad de París — más conocida como La Sorbona-, Francois-Guy Trebulle (Fernández, 2018), entre otros, coinciden en apuntar que las universidades están en peligro de desaparecer si no se adaptan a las particularidades del actual escenario. Esta advertencia o pronóstico - más allá de que se materialice - redunda en la necesidad de repensar la educación a partir de las particularidades y las transformaciones de los diferentes actores y procesos que poseen un protagonismo destacado en el mismo: el perfil de alumnado, las metodologías docentes, el rol del profesorado y el tipo de evaluación aplicada para certificar la adquisición de habilidades y competencias. 
Desde que McLuhan pronosticara en 1960 la irrupción de "aula sin muros", diversos autores, como Stinson (1997), Prensky (2007), Piscitelli, Adaime y Binder (2010), Pastor (2010), Pérez-Tornero, Tejedor, Simelio y Marín (2015), entre otros, han incidido en la necesidad de reformular los procesos formativos con el objetivo de incrementar el componente lúdico, motivador e inmersivo de los mismos, especialmente, en el marco de la denominada sociedad-red (Castells, 2003). Este conjunto de trabajos se ha desarrollado en una sociedad impactada por la cibercultura (Lévy, 2007) y por la esencia líquida (Bauman, 2003) de un escenario marcado por el protagonismo progresivo que ha adquirido el componente tecnológico y, especialmente, las redes sociales (Pempek et al., 2009; Tuñez, \& Abejón, 2010), que han llegado a generar nuevos hábitos entre los cibernautas (Waycott et al., 2010). Este conjunto de cambios ha ido unido a las transformaciones de la web, que de su versión 1.0 ha ido ascendiendo a los estadios de web social o 2.0, web semántica o 3.0 y web de los objetos o 4.0. La idiosincrasia colaborativa, social, participativa y bidireccional de la web 2.0 supuso un impacto hondo en el escenario educativo. Como apuntan Castaño et al. (2008) esta versión renovada del ciberespacio impacta directamente (Ver Tabla 1) en los procesos educativos, al tiempo que inaugura interesantes y provechosas oportunidades de innovación que afectan a los roles de los diferentes actores (profesores y alumnos, especialmente), a los contenidos y a las metodologías, entre otros aspectos.

\section{Tabla 1}

\section{Principales transformaciones del escenario educativo}

\begin{tabular}{|l|l|}
\hline Producción individual de contenidos & $\begin{array}{l}\text { Profesores y alumnos como creadores activos del } \\
\text { conocimiento. }\end{array}$ \\
\hline Aprovechamiento del poder de la comunidad & Aprender con y de otros usuarios. \\
\hline $\begin{array}{l}\text { Arquitectura de la participación de los } \\
\text { servicios }\end{array}$ & Todos pueden ser actores activos. \\
\hline $\begin{array}{l}\text { Potenciación de herramientas sencillas e } \\
\text { intuitivas }\end{array}$ & El usuario no ha de ser un técnico especialista. \\
\hline Apuesta por el software abierto & Filosofía de “apertura" a los estándares no propietarios. \\
\hline Creación de comunidades de aprendizaje & $\begin{array}{l}\text { Usuarios comprometidos por intereses, temáticas, } \\
\text { etc. compartidas. }\end{array}$ \\
\hline Efecto Red & $\begin{array}{l}\text { Del trabajo individual a la cooperación entre } \\
\text { iguales. }\end{array}$ \\
\hline
\end{tabular}

Fuente: Castaño et al. (2008). 
En este contexto, los denominados "milennial" (Francese, 2003), cuyo perfil se aproxima al del "prosumidor" acuñado por Toffler (1980), dan forma a un alumnado que, tanto en su acceso a la información (Akter, \& Nweke, 2016) como en sus expectativas profesionales y en su quehacer cotidiano confiere una gran importancia a las tecnologías. Esta "Generación del Milenio" o "Generación Y" la integran usuarios que destacan por su perfil multitarea, por su capacidad intuitiva en el manejo de los ecosistemas digitales (Ruano et al., 2016) y por poseer una atención que se ha definido como "flotante" (Crenshaw, 2010). Varias investigaciones han diagnosticado una pérdida de interés o distanciamiento de estos alumnos respecto a los contenidos y las dinámicas de trabajo propios del actual sistema educativo. En España, según un estudio del catedrático de Sociología, Mariano Fernández Enguita, uno de cada tres alumnos se aburre en la escuela y abandona los estudios (Fernández, 2016). Este tipo de datos se producen en un escenario marcado por un conjunto de cambios tecnológicos que afectan a los perfiles profesionales (Lin, 2012).

En el ámbito de la comunicación, las demandas formativas han sido diversas. Para Pavlik (2005), la innovación tecnológica ha modificado los estudios de periodismo en cuatro grandes áreas: la manera de enseñar e investigar; el contenido de lo que enseñamos, la estructura de los departamentos de periodismo y de las facultades de comunicación y las relaciones entre los docentes y sus públicos (estudiantes, financiadores y competidores). Autores como Pavlik (2005), Tarcia y Marinho (2008), García Avilés y Martínez, (2009), López (2010), Roblyer (2010), López García (2012), Silva (2014), Singh (2015), Pérez-Tornero y Varis (2012), Pérez-Tornero (2016), Sánchez, Campos y Berrocal (2015), Tramullas (2016), Sánchez-García (2017), Tejedor y Cervi (2017), Tejedor, Giraldo-Luque y Carniel (2018) han planteado en diferentes trabajos la importancia y la urgencia de repensar el enfoque de los planes de estudio (tanto en su oferta curricular como en sus metodologías) del escenario de la comunicación. En el campo del periodismo, un estudio realizado a mediados de 2006 en España concluía que la mayoría de las facultades no lograban responder a las necesidades formativas demandadas por la industria (Tejedor, 2007).

La Expedición Tahina-Can es un proyecto que, desde el año 2004, organiza un viaje académico a diferentes enclaves del mundo en el que participan predominantemente estudiantes universitarios del ámbito de comunicación. Organizados en equipos de prensa, fotografía y televisión, los expedicionarios participan en talleres de producción de contenidos, debates y otras actividades que, en todos los casos, se apoyan en la metodología del 
aprender haciendo a través de un estrecho vínculo con la cotidianeidad y las historias de vida de los lugareños de los países visitados. El proyecto nació como una respuesta a la necesidad de idear nuevos escenarios y nuevas metodologías de aprendizaje, especialmente, en el ámbito del periodismo y la comunicación donde los contenidos se han transformado en unidades portátiles, personalizadas y participativas (Matsa, \& Mitchell, 2014).

Partiendo de este escenario, la investigación, basada en un estudio de caso, ha analizado las posibilidades del viaje como recurso educativo a partir de las catorce ediciones (2004-2018) de la Expedición Tahina-Can, un proyecto que organiza el Departamento de Periodismo y Ciencias de la Comunicación de la Universidad Autónoma de Barcelona, a través del grupo de investigación del Gabinete de Comunicación y Educación. El estudio responde a las siguientes preguntas de investigación de ámbito descriptivo:

- ¿Cuáles son las fortalezas de la expedición como proyecto formativo?

- ¿Cuáles son sus debilidades o aspectos a mejorar?

- ¿Cómo los alumnos participantes (del ámbito de la comunicación) valoran la experiencia a nivel formativo?

\section{El viaje como recurso educativo}

La idea de que el viaje es fuente de educación continua vigente y puede entenderse como una práctica constructivista (Vygotsky, 1978). La sistematización del viaje como currículo se manifiesta a través de diversas iniciativas que no sólo recogen el valor formativo del intercambio cultural, sino que en ellas se propone además una educación en valores basada en la responsabilidad, el compromiso y la comprensión hacia otros países y culturas. Una experiencia vital integrada por equipos interdisciplinarios en la que se produce un antes, un durante y un después muy intensos.

Bajo el paradigma de que el conocimiento se construye, el modelo constructivista interpreta al viaje como un proceso de aprendizaje basado en la construcción autónoma del conocimiento. Por una parte, permite la asimilación de nuevos conocimientos con ejercicios en los que los sujetos activos dan significados a nuevas experiencias (Blashki, \& Nichol, 2008). Por otra parte, el proceso de aprendizaje genera estados de desequilibrio - en su enfrentamiento a nuevos contextos en los que el viajero se enfrenta a escenarios desconocidos - que exigen del sujeto momentos de reacomodación que propi- 
cian cambios en las estructuras de pensamiento y promueven la asignación de significados a través de experiencias (Medina Gallego, 2003, pp. 115-162).

Piaget (1973), Kohlberg (1992), Ausubel y Sullivan (1983) y Vygotsky (1978) coinciden en que la construcción del conocimiento se realiza desde las estructuras cognitivas individuales. La teoría constructivista señala que esa estructuración se propicia desde espacios cotidianos como el hogar, la escuela, la sociedad. En el viaje, la cotidianidad adquiere la forma de la convivencia continua, durante un periodo de tiempo, con el propio espacio cotidiano de la experiencia que se vive. El constructivismo retoma las experiencias directas del viajero y las reorganiza para, con la experiencia del viaje, generar un cambio conceptual que propicia el aprendizaje significativo (Ausubel, \& Sullivan, 1983). Mediante la confrontación de hipótesis y de métodos de resolución de problemas y de su observación el individuo realiza de forma constante un diagnóstico entre su propio nivel y el nivel potencial de desarrollo, siendo el diagnóstico el ámbito de aplicación de la "zona de desarrollo próximo" (Vygotsky, 1978). La relación establecida como marco de aprendizaje hacia un nivel más alto del conocimiento refuerza los aportes propios del viajero en su desarrollo cognitivo. Durante el viaje, la adquisición de conocimientos será un proceso en el que las nuevas informaciones serán incorporadas a los esquemas y estructuras preexistentes, modificándolas.

Al mismo tiempo, el constructivismo señala que el conocimiento no es una copia fiel de los fenómenos externos. Es una construcción del ser humano. Lo que pretende el marco del aprendizaje es la ordenación de la experiencia de tal forma que su secuencialización permita realizar una distinción entre lo externo y lo interno - al individuo o al grupo - para crear una nueva realidad (Medina Gallego, 2003, pp. 163-189). El constructivismo reconoce que existe un sujeto activo que llega al proceso educativo con una visión de mundo que influye sobre su forma de actuar. Por ello, otorga al aprendizaje una necesidad de producción del cambio individual (actitudinal, metodológico, axiológico, estético) y una proyección hacia la construcción de escenarios que faciliten dichos cambios.

El aprendizaje constructivista se preocupa por el desarrollo de conocimientos útiles, no de aprender por aprender. De acuerdo con su lógica, los nuevos saberes deben poder aplicarse en el mundo de forma práctica (Doolittle, \& Camp, 1999). El aprendizaje constructivista encuentra así, en el viaje, un contexto auténtico para poder mostrar a los alumnos la aplicación absolutamente práctica de aquello que aprenden. Además, los estudiantes 
necesitan trabajar en equipo e interactuar con sus compañeros para poder comprender conceptos a través de la construcción de conocimientos obtenidos con experiencias del propio viaje. Las prácticas relacionadas con la reflexión aumentarán las habilidades metacognitivas de los estudiantes.

Las teorías constructivistas asumen, por tanto, que los viajeros-aprendices crean representaciones mentales de su entorno mediante el uso de su conocimiento en contextos reales (Doolittle, \& Camp, 1999; Driscoll, 2005; Mayer, 1999). Mediante las experiencias personales, las interacciones sociales y la metacognición, los participantes del viaje adquieren la capacidad de pensar de forma crítica, de razonar y de desarrollar la comprensión (Driscoll, 2005). En el viaje, el modelo constructivista propone un cambio conceptual en dos dimensiones: un cambio semántico, pues se amplían los conocimientos al producir una crisis en las ideas previas de los viajeros con la intención de que sean revaluadas, y un cambio teórico, debido la revisión de los conocimientos anteriores que produce un cambio de ideas sobre los fenómenos objeto de conocimiento. Dentro del constructivismo se promueve la insatisfacción frente a teorías iniciales o ideas previas, y se promueve la integración de nuevas teorías explicativas basadas en la experiencia, el viaje, que servirán para falsear la teoría anterior (Medina Gallego, 2003, pp. 275-323) y superarla.

El marco constructivista descrito, dentro de un escenario educativo del siglo XXI, puede también ser englobado bajo la conceptualización de una competencia entendida como la capacidad de resolución de problemas. Para Cano (2008), quien propone una serie de características que engloban el concepto de competencia a partir de una revisión de más de cincuenta definiciones de diferentes autores, una competencia a) está integrada por habilidades o capacidades relacionadas con el saber hacer (acciones) de un individuo, b) requiere de un proceso de acción reflexiva por parte del sujeto que las realiza (acción+ reflexión), c) es adquirible a través de diferentes procesos (aprendizaje) y, en tal sentido, es actualizable, d) permite resolver situaciones problemáticas en un contexto específico de forma eficiente, autónoma y flexible, y e) tiene en su ejecución, de acuerdo a los factores anteriores, respuestas individualizadas o diversas atendiendo a los factores contextuales específicos.

\section{Metodología}

El desarrollo del artículo se ha planteado como un estudio de caso cualitativo y centrado en un objeto concreto: la Expedición Tahina-Can como ejem- 
plo de viaje educativo. Al mismo tiempo, el análisis complementa su enfoque cualitativo a través del desarrollo de un focus group que permite corroborar los planteamientos teóricos con la visión que participantes de la Expedición tienen sobre su propia concepción del viaje vivido como experiencia educativa.

Los estudios de caso son definidos como un método para aprender sobre una instancia compleja (US General Accounting Office, 1990), a partir de la comprehensión y descripción de ella como un todo y su contexto, mediante datos e información obtenidos por descripciones extensivas (Mertens, 2005). Gillham (2000) señala que el estudio de caso detalla una unidad relacionada con actividades humanas que se desarrollan en el mundo real, entendida en su contexto, que existe en el presente y que se mezcla con el contexto de tal manera que resulta complicado precisar sus fronteras.

Gerring, para quien los diseños de investigación del caso pueden abarcar una o varias unidades de estudio, (2007) aprecia al estudio de caso como un trabajo cuyo método es cualitativo y holístico, y que ofrece un examen complejo y amplio de un fenómeno, que utiliza un tipo de evidencia particular y que usa la triangulación de datos. Según Hernández et al. (2006) el método puede definirse como un estudio que puede utilizar procesos de investigación cualitativa y que analiza en profundidad una unidad para responder al planteamiento del problema, probar hipótesis o desarrollar teorías.

El estudio de caso aplicado a presente investigación toma como unidad de análisis a la Expedición Tahina-Can y se define, por tanto, como cualitativo y sincrónico. El análisis del caso utiliza la descripción como fundamento metodológico, en la que se incluyen la contextualización del programa, la construcción del plan académico y el impacto mediático obtenido en sus ediciones.

El estudio de caso se complementa y se triangula con el desarrollo de un grupo focal en el que intervinieron cinco estudiantes que habían participado en ediciones pasadas de la Expedición Tahina-Can. El desarrollo del focus group tuvo el objetivo de promover una conversación profunda sobre la experiencia del viaje de los participantes en las ediciones descritas de la Expedición. El método se concibe como un espacio de interacción y diálogo entre personas que comparten una serie de características comunes y que conduce a la construcción grupal de significados, a partir de la narrativa colectiva de los participantes (De Miguel en Berganza, \& Ruiz, 2005, p. 269). Los resultados del grupo focal fueron utilizados para comprobar los argumentos teóricos expuestos con anterioridad, en los que se concibe al viaje como una 
experiencia de aprendizaje construccionista, significativo y de alto interés para sus participantes.

\section{La expedición Tahina-Can}

La Expedición Tahina-Can es un proyecto de periodismo de viajes que organiza el Gabinete de Comunicación y Educación de la Universidad Autónoma de Barcelona, dirigido por el catedrático José Manuel Pérez Tornero, con el objetivo de promover una manera diferente y genuina de entender y llevar a cabo los viajes. El proyecto, premiado en 2004 con el primer premio del II Certamen Internacional Intercampus sobre Investigación y Docencia en la Red 2004 organizado por la Fundación Telefónica, en la categoría de “Aprendizaje en la Red", pretende que jóvenes universitarios - de diferentes carreras y universidades - emprendan un viaje iniciático que les enfrente a realidades socio-culturales diferentes a las suyas y que "eduque" su manera de ver y explicar lo visto y lo vivido. En el marco de la Expedición, los participantes se organizan en equipos de prensa, radio, televisión y fotografía; y deben producir relatos viajeros que huyan de lo convencional o turístico en aras de producir "mensajes" críticos que inviten a la reflexión desde la alteridad.

El proyecto que empezó en el año 2004 pretende fomentar el interés de la comunidad universitaria por la cultura y las costumbres del país visitado. Tras catorce ediciones en activo, la iniciativa promueve fuertes vínculos entre los expedicionarios y los estudiantes, instituciones y medios de comunicación de cada destino. Se trata, sobre todo, de una propuesta que pretende instaurar una nueva concepción de los viajes orientada hacia el compromiso, la compresión y el análisis hacia el destino recorrido. Esta aproximación al periodismo, la aventura y la cooperación internacional se concreta en un conjunto de líneas temáticas como son el conocimiento de otras realidades socio-culturales, el conocimiento también de cómo funcionan los medios de comunicación del país en cuestión y la participación en el desarrollo de distintos proyectos periodísticos mediante la producción durante el viaje de contenidos periodísticos de diferente naturaleza.

A lo largo de casi dos semanas, los expedicionarios recorren un país del mundo, estudiando las particularidades del panorama cultural y mediático a través de un itinerario que se aleja de los circuitos turísticos tradicionales. 
Cada año, Tahina-Can convoca a estudiantes españoles de todas las titulaciones universitarias con el propósito de crear un perfil multidisciplinar.

Tabla 2

Listado de ediciones de la Expedición Tahina-Can

\begin{tabular}{|l|c|}
\hline \multicolumn{1}{|c|}{ Expedición } & Año \\
\hline México: Viaje al mundo maya & 2004 \\
\hline Rumbo al ecuador: De Quito al Amazonas & 2005 \\
\hline República Dominicana: Por tierra de taínos. La puerta al nuevo mundo & 2006 \\
\hline Cuba: Una isla en su encrucijada & 2007 \\
\hline Perú: Rumbo al mundo inca - Cooperación en los andes & 2008 \\
\hline Ecuador: De la mitad del mundo al corazón del Amazonas & 2009 \\
\hline Chile: Crónica de la reconstrucción & 2010 \\
\hline Marruecos: Viaje al reino del lejano poniente & 2012 \\
\hline Tailandia: Aventura en el reino del Siam & $2013-2014$ \\
\hline Uzbekistán: A Samarcanda por la Ruta de la Seda & 2015 \\
\hline República Dominicana: La aventura de la educación & 2016 \\
\hline Colombia: Tributo a la paz - En busca de la ciudad perdida & 2017 \\
\hline Yucatán: Tesoros del mundo maya & 2018 \\
\hline
\end{tabular}

Fuente: Elaboración propia

\section{El programa académico}

La Expedición Tahina-Can gira en torno a un programa académico producido por el equipo que dirige el proyecto, y cuya elaboración, previa al viaje, cuenta además con la asesoría de docentes, periodistas y profesionales del mundo de la comunicación del país de destino en cuestión. Esta experiencia de ciberperiodismo de viajes se construye de acuerdo al objetivo de estrechar las relaciones de cooperación entre los universitarios españoles y los estudiantes, instituciones y medios de comunicación del país visitado, mediante el análisis y estudio de las particularidades del panorama cultural y mediático que presentan. En este sentido, los expedicionarios se adentran en otras realidades socio-culturales, conocen el funcionamiento de sus medios de comunicación y participan en el desarrollo de proyectos periodísticos. A lo largo del viaje, el programa se articula según las actividades previstas entre las que destacan charlas, seminarios, conferencias y coloquios sobre as- 
pectos ligados a la actualidad del lugar e impartidas por periodistas especializados de diferentes medios, entrevistas con profesionales de los medios de comunicación, elaboración de reportajes y otro tipo de productos periodísticos. Los participantes, por su parte, tienen la oportunidad de informar del transcurso de la Expedición a través de la página web del proyecto con sus crónicas, entrevistas y otros materiales (Ver Gráfico 1). Los expedicionarios aprenden de un país, de sus gentes, de su historia, de su folclore, de su gastronomía y también de sus problemas, de sus fortalezas, de sus necesidades, de sus contradicciones. Especialmente, aprenden la importancia de situarse en el lugar del "otro". Esto significa aprender a "mirar" y procesar nuestro entorno y nuestro mundo. Aprenden a reflexionar sobre sus prioridades y su escala de valores, y ponerlas en entredicho. Aprenden también de uno mismo y de su vocación profesional y humana.

\section{Gráfico 1}

\section{Página web de la Expedición Tahina-Can}

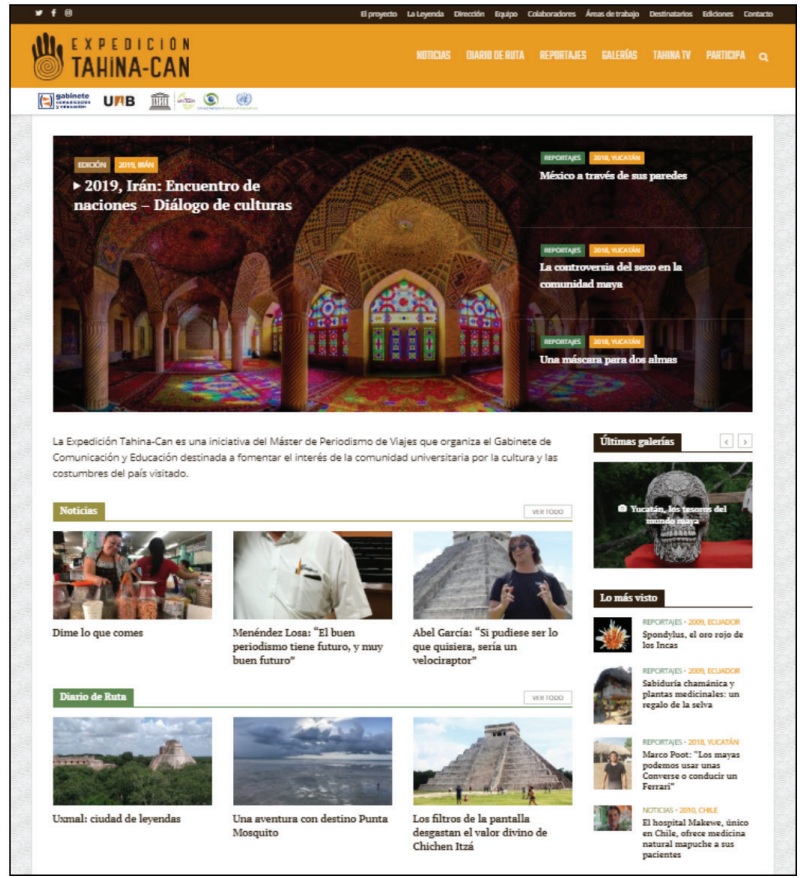

Fuente: www.tahina-can 
Existen diferentes talleres que, en todos los casos, pretenden que los expedicionarios se ejerciten en la producción sobre el terreno de contenidos periodísticos a partir de la utilización de las técnicas y rutinas de producción periodísticas y del aprovechamiento de los diferentes atributos informativos que poseen a su alcance (Ver Gráfico 2).

\section{Gráfico 2}

\section{Ejemplo de reportaje elaborado por expedicionarios}

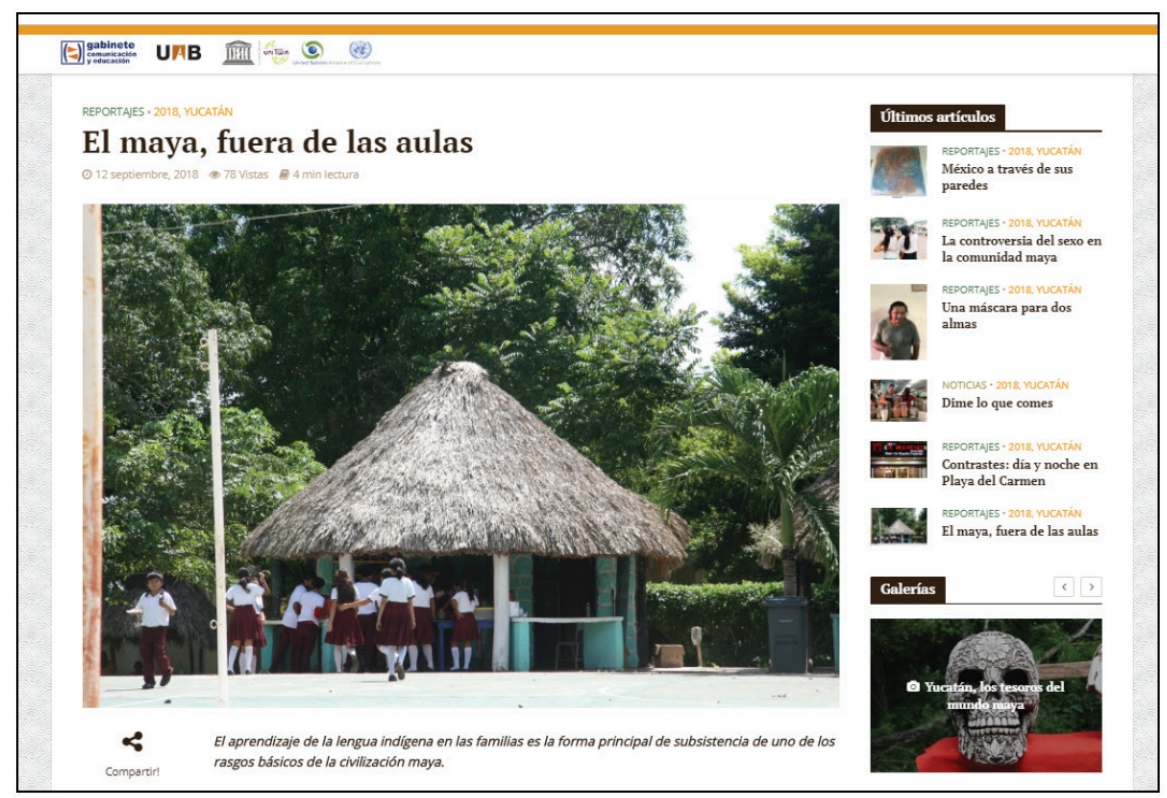

Fuente: www.tahina-can

Finalizado el viaje, el equipo del Gabinete de Comunicación y Educación organiza el acto de presentación de los diferentes trabajos periodísticos elaborados por los estudiantes en los talleres de prensa, fotografía, radio y televisión. El acto tiene lugar cada año y se celebra en una universidad española. Como complemento, se realiza también una exposición fotográfica itinerante por España, donde se exponen las fotografías más representativas realizadas por los propios expedicionarios. El proyecto se completa con la creación de un libro de viajes centrado en el país visitado y concebido con 
una filosofía diferente. Se trata de una obra en la que se incluyen, principalmente, las crónicas de los expedicionarios, los datos socio-culturales así como los artículos de periodistas especializados en viajes.

Gráfico 3

Noticia sobre el proyecto difundida en medio

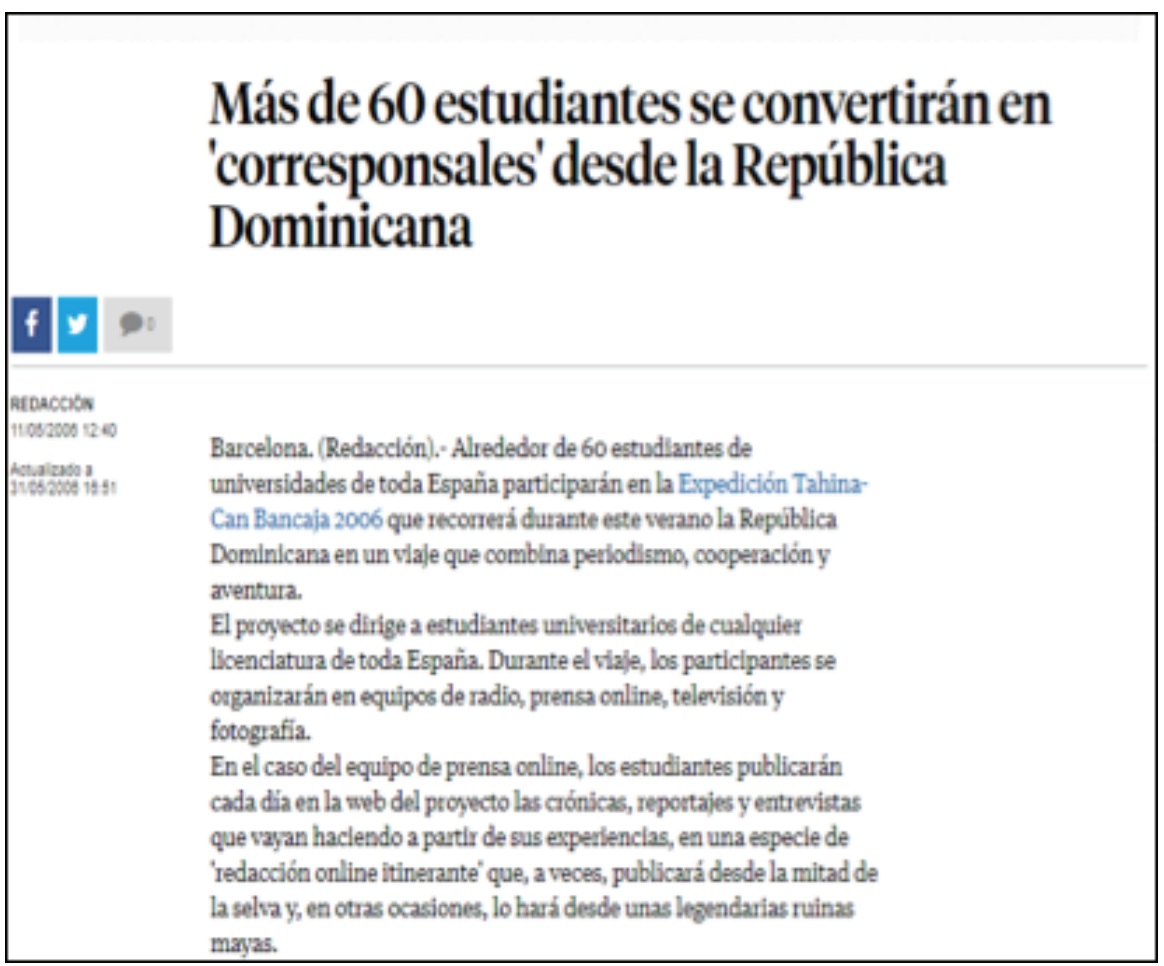

Fuente: www.lavanguardia.com

\section{Discusión}

La descripción del caso estudiado, la Expedición Tahina-Can como experiencia de viaje educativo, demuestra - tanto en los discursos teóricos sobre el modelo educativo construccionista y sobre el nuevo tipo de perfil de estudiante de la actualidad, como en las propias narraciones de los estudian- 
tes evidenciadas en el grupo focal realizado - una cercanía evidente entre el programa educativo ofertado en el viaje y los conceptos fundamentales que constituyen el cambio pedagógico orientado a las necesidades formativas del siglo XXI.

En el grupo focal uno de los estudiantes señalaba que "el viaje realizado me ha permitido alejarme de la zona de confort en la que normalmente me encuentro, propiciando un choque entre mi propia realidad y la nueva realidad explorada". El desarrollo de estados de desequilibrio o reacomodación entre conocimientos o imaginarios previos y los nuevos contextos de aprendizaje es uno de los ejes del ejercicio constructivista pues promueve la asignación de significados y la construcción de nuevos conceptos a través de la experiencia viva (Medina Gallego, 2003).

Para los estudiantes, Tahina-Can es un viaje educativo porque "cada día y cada actividad implica una experiencia de aprendizaje", o porque "tenemos una evolución desde el primer día de trabajo y convivencia que se proyecta incluso después del viaje". Para alguno de ellos el viaje representa un punto "más allá del nivel académico que tenemos como idea de la educación". El cambio de perspectiva sobre su propia experiencia de aprendizaje, y el cambio interiorizado por los viajeros, denota la confrontación de hipótesis previas a partir de una vivencia y del diagnóstico cotidiano individual y grupal sobre el alcance y superación de las "zonas de desarrollo próximo" definidas por Vygotsky (1978). La nueva información, ligada a una recepción también emocional y no solamente racional, se incorpora - no sin un reacomodo cognitivo - a las estructuras y esquemas previamente construidas en el estudiante. En el proceso del viaje, como demuestran los estudiantes participantes en el grupo focal, los esquemas previos terminan por modificarse. Los viajeros, por tanto, asisten a las dos dimensiones del cambio conceptual presentadas por Medina Gallego (2003). De una parte, realizan una transformación semántica al revaluar sus propios imaginarios sobre una realidad que acaban por conocer de forma directa y sin intermediarios. Por otra parte, generan un cambio teórico al propiciar la revisión propia de sus explicaciones. El viaje demuestra la insatisfacción frente a teorías iniciales o ideas previas y facilita la creación de nuevas explicaciones y descripciones sociales de la realidad vivida.

Al mismo tiempo, la necesidad de concebir al estudiante como sujeto autónomo del proceso de aprendizaje (Blashki, \& Nichol, 2008) se hace presente en la vivencia como un marco de posibilidades para dar significado a 
nuevas experiencias: "la Expedición se me presentó como una oportunidad para conocer y para descubrir mis propias curiosidades. Para aprender a partir de mis propios intereses", indicaba otro de los estudiantes participantes en el grupo focal. McLoughlin y Lee (2008), así como Robinson (2006), señalan a los conceptos de curiosidad y de creatividad como principios activos del interés del estudiante hacia su propio contexto de aprendizaje y hacia la resolución de los problemas propuestos en el proceso educativo.

Algunos estudiantes mencionaron también que las prácticas periodísticas desarrolladas durante la Expedición:

No eran entendidas por nosotros como un trabajo formal de la Universidad. En la experiencia del viaje nos obligábamos a buscar historias y a preguntar de verdad. Éramos conscientes de que los productos que realizábamos saldrían del aula, que serían publicados. Teníamos una motivación extra en la concepción de nuestro ejercicio periodístico como una práctica real.

El testimonio implica también una conexión muy clara con el sentido práctico y con la aplicación real del conocimiento a un contexto particular definido por el modelo construccionista (Doolittle, \& Camp, 1999). Los estudiantes logran desarrollar, así, una buena parte de las siete necesidades competenciales descritas por Nightingale y O'Neil (1994) a través de un trabajo absolutamente práctico. Al mismo tiempo, se hace palpable la relación entre los objetivos curriculares y proyectos reales desarrollados durante el viaje (Trilling, \& Fadel, 2009): "en la Expedición logramos mejorar nuestras habilidades en aspectos técnicos de la producción periodística, al tiempo que hicimos propias las rutinas productivas propias de cada uno de los medios en los que trabajamos", manifestaba un estudiante.

El conocimiento construido en el contexto real, a través de experiencias prácticas (Doolittle, \& Camp, 1999; Driscoll, 2005), ayuda a los estudiantes a comprender mejor la propia realidad. Cada uno de ellos crea una representación mental a partir de su experiencia vital. Su cercanía con el contexto y la realidad, guiada por el planteamiento de problemas y por el trabajo colaborativo, permite también la formación de un pensamiento crítico y el desarrollo de procesos más complejos de comprensión y de racionalización (Driscoll, 2005). 


\section{Conclusiones}

Las catorce ediciones del proyecto de la Expedición Tahina-Can permiten extrapolar una serie de conclusiones sobre el potencial del viaje como práctica constructivista que asume los retos del perfil del estudiante del siglo XXI. El análisis de las particularidades del proyecto, a través de la metodología del estudio de caso junto a los resultados derivados de los grupos focales desemboca en las siguientes conclusiones:

El viaje como aventura de exploración y descubrimiento: El estudio desarrollado y las experiencias derivadas de las diferentes ediciones del proyecto permiten afirmar que el viaje constituye una sólida experiencia de exploración y descubrimiento para los expedicionarios que no se limita a la Expedición, sino que se proyecta en el post viaje. Los expedicionarios manifiestan que tras la experiencia vivida poseen un mayor interés por aspectos de su cotidianeidad que anteriormente no les había resultado de interés.

El componente vivencial como estímulo sobresaliente: La combinación de un programa académico junto a la experiencia de visitar un país (a través de diferentes visitas: culturales, medioambientales, deportivas, etc.) constituye un gran estímulo para los participantes que, según ha constado en la investigación, destacan especialmente lo importante de realizar una cobertura periodística sobre el terreno entablando un contacto directo con la realidad. El alumnado participante se confesaba muy estimulado por los encargos que recibía durante la Expedición, al tiempo que manifestaba una gran motivación para esforzarse en aras de generar contenidos que se publicarían en el sitio web del proyecto y que serían visionados y comentados por sus propios compañeros de viaje.

El viaje como dinámica de roles periodísticos: El proyecto se consolida como un escenario idóneo para la aplicación por parte del alumnado de las rutinas de producción periodísticas propias de la prensa, la radio, la televisión y la fotografía. Además, se detecta una tendencia a la convergencia entre los equipos o talleres de trabajo, que viene dada no solo por el intercambio de contenidos sino también por la concepción y desarrollo colaborativo de coberturas informativas y reportajes a partir de los aportes de los integrantes de diferentes equipos. De este modo - se observa - por un lado, una familiarización e identificación con la lógica transmedia y, por otro, se confirma la eficacia de la Expedición para potenciar una competencia decisiva en los comunicadores como es el trabajo en equipo. 
Finalmente, el estudio del proyecto junto a las reflexiones del alumnado advierte igualmente de una serie de aspectos que deberían ser objeto de remodelación o mejora en el marco de esta iniciativa o de proyectos similares que ubiquen el viaje como motor de un proceso formativo. Por último, partiendo de esta investigación, se plantea la importancia de consolidar y proyectar esta línea de investigación en la que convergen la educación, los viajes y la comunicación por su interés y pertinencia en el actual escenario educativo.

\section{Agradecimientos}

Este estudio ha contado con la colaboración de las investigadoras Judith Martínez, Gisela Martínez y Laura Meneses.

\section{Bibliografía}

Akter, T. \& Nweke, G.E. (2016). Social media users and their social adaptation process in virtual environment: Is it easier for Turkish Cypriots to be social but virtual beings? Computers in Human Behavior, 61, 472-477. https:// doi.org/10.1016/j.chb.2016.03.067

Amiguet, L. (07/09/2017). Muchas universidades van a cerrar porque no hacen falta. La Vanguardia. Recuperado de https://goo.gl/APU1HF. (4-10-2018).

Ausubel, D.P., \& Sullivan, E.V. (1983). El desarrollo infantil. Barcelona: Paidós. Bauman, Z. (2003). Modernidad líquida. México: Fondo de Cultura Económica.

Berganza, M. R., \& Ruiz, J. (2005). Investigar en comunicación: guía práctica de métodos y técnicas de investigación social en comunicación. Madrid: McGraw-Hill.

Cano, E. (2008). La evaluación por competencias en la educación superior. Profesorado. Revista de currículum y formación del profesorado, 12(3), 1-16. Recuperado de https://goo.gl/73HhPZ (5-11-2018).

Castaño, C.M., Maiz, I., Palazio, G.J., \& Villarroel, J.D. (2008). Prácticas educativas en entornos web 2.0. Madrid: Síntesis.

Castells, M. (2003). La galaxia Internet. Barcelona: Mondadori.

Crenshaw, D. (2010). The Myth of Multitasking: How 'Doing It All' Gets Nothing Done. San Francisco: Jossey-Bass.

Doolittle, P.E., \& Camp, W. G. (1999). Constructivism: The career and technical education perspective. Journal of Career and Technical Education, 16(1), 23-46. Recuperado de https://goo.gl/2tirdm (14-10-2018). 
Driscoll, M.P. (2005). Psychology of learning for instruction. Boston, MA: Pearson Education, Inc.

Farreras, C. (23/04/2016). La mitad de las universidades desaparecerán en diez años. La Vanguardia. Recuperado de https://goo.gl/tB4tjU (5-5-2018).

Fernández Enguita, M. (2016). La educación en la encrucijada. Madrid: Fundación Santillana.

Fernández, M. (02/06/2018). Si las universidades no evolucionamos, vamos a desaparecer. Infobae. Recuperado de https://goo.gl/tQ5NBE (10-6-2018).

Francese, P. (2003). Trend Ticker: Ahead of the Next Wave. Recuperado de https://bit.ly/1vfypdp (29-5-2018).

García Avilés, J.A., \& Martínez, O. (2009). Competencias en la formación universitaria de periodistas a través de nuevas tecnologías. Revista de la Facultad de Ciencias Sociales y Jurídicas de Elche, 1(4), 239-250. Recuperado de https://goo.gl/w1twh9 (10-11-2018).

Gerring, J. (2007). Case Study Research. Principles and practices. Nueva York: Cambridge University Press.

Gillham, B. (2000). Case study research methods. London: Continuum.

Hernández, R; Fernández, R., \& Baptista, P. (2006). Metodología de la investigación. Bogotá: McGraw-Hill.

Kohlberg, L. (1992). Sicología del desarrollo moral. Bilbao: Desclée de Brouwer S.A. Lévy, P. (2007). Cibercultura: la cultura de la sociedad digital. Barcelona: Anthropos. Lin, M. (22/06/2012). The gap between journalism education and the (changing) journalism profession. MulinBlog: A digital journalism blog. Recuperado de https://goo.gl/JuHpKW (22-4-2018).

López, X. (2010). La formación de los periodistas en el siglo XXI en Brasil, España, Portugal y Puerto Rico. Revista Latina de Comunicación Social, 65, 231-243.

López, X. (2012). La formación de los periodistas para los entornos digitales actuales. Revista de Comunicación, 11, 178-195. Recuperado de https://goo. $\mathrm{gl} / 5 \mathrm{Zt} 2 \mathrm{cN}(12-11-2018)$.

Matsa,K.E.\& Mitchell,A. (2014). 8 Keys Takeaways about Social Media and News. Pew Research Center. Journalism \& Media, 26 marzo. Disponible en: https://goo.gl/mPUxHj (12-6-2018).

Mayer, R.E. (1999). Designing instruction for constructivist learning. En C. Reigeluth, (Ed.), Instructional-design theories and models (pp. 141-159). Mahwah: Lawrence ErlbaumAssociates. 
McLoughlin, C., \& Lee, M. (2008). The Three P's of Pedagogy for the Networked Society: Personalisation, participation, and productivity, International Journal of Teaching and Learning in Higher Education, 20(1), 10-27. Recuperado de https://goo.gl/voxEGz (1-11-2018).

Mertens, D.M. (2005). Research and evaluation in Education and Psychology: Integrating diversity with quantitative, qualitative and mixed methods. Thousand Oaks: Sage.

Medina Gallego, C. (2003). Didáctica para la transformación de la vida escolar. Bogotá: Colciencias, IPAC, Fundación Social, Universidad Nacional.

Nightingale, P. \& O’Neil, M. (1994). Achieving Quality Learning in Higher Education. Londres: Kogan Page.

Pastor, L. (2010). Funiversity. Los medios de comunicación cambian la universidad. Barcelona: Editorial UOC.

Pavlik, J. (2005). El periodismo y los nuevos medios de comunicación. Barcelona: Paidós.

Pempek, T.A., Yermolayeva, Y.A., \& Calvert, S.L. (2009). College students' social networking experiences on Facebook. Journal of Applied Developmental Psychology, 30(3), 227-238. https://doi.org/10.1016/j.appdev.2008.12.010

Pérez-Tornero, J.M. \& Varis, T. (2012). Alfabetización mediática y nuevo humanis$m o$, Barcelona: UNESCO - UOC Press.

Pérez-Tornero, J.M., Tejedor, S., Simelio, N., \& Marín, B. (2015). Estudiantes universitarios ante los retos formativos de las Redes Sociales: el caso de Colombia. Estudios sobre el Mensaje Periodístico, 21(1), 509-521. doi: 10.5209/rev_ESMP.2015.v21.n1.49108

Pérez-Tornero, J.M., \& Tejedor, S. (Eds.) (2016). Ideas para aprender a aprender. Manual de innovación educativa y tecnología. Barcelona: Editorial UOC.

Piaget, J. (1973). La representación del mundo en el niño. Madrid: Morata.

Piscitelli, A., Adaime, I., \& Binder, I. (Comp.) (2010). El Proyecto Facebook y la posuniversidad. Sistemas operativos sociales y entornos abiertos de aprendizaje. Madrid: Fundación Telefónica, Ariel.

Prensky, M. (2007). Digital Game-Based Learning. Minnesota: Paragon.

Robinson, K. (2006) “Do Schools Kill Creativity?" Ted Talks. Recuperado de https://goo.gl/UV3uLa (4-5-2018).

Roblyer, M., McDaniel, M., Webb, M., Herman, J., \& Witty, J. (2010). Findings on Facebook in higher education: A comparison of colleague faculty and student 
uses and perceptions of social networkings sites. The Internet and Higher Education, 3(13), 134-140. https://doi.org/10.1016/j.iheduc.2010.03.002

Ruano, L.E., Congote, E.L., \& Torres, A.E. (2016). Comunicación e interacción por el uso de dispositivos tecnológicos y redes sociales virtuales en estudiantes universitarios. Revista Ibérica de Sistemas y Tecnologías de Información, 19, 15-31. http://dx.doi.org/10.17013/risti.19.15-31

Sánchez-García, P., Campos, E., \& Berrocal, S. (2015). Las funciones inalterables del periodista ante los perfiles multimedia emergentes. Revista Latina de Comunicación Social, 70, 187-208. https://doi.org/10.4185/RLCS-2015-1042

Sánchez-García, P. (2017). Periodistas (In)formados. Un siglo de enseñanza periodística en España: historia y tendencias. Madrid: Universitas.

Stinson, S. (1997). A question of fun: adolescent engagement in dance education. Dance Research Journal, 29(2), 49-69. https://doi.org/10.2307/1478734

Tarcia, L., \& Marinho, S. P. (2008). Challenges and New Ways of Teaching Journalism in Times of Media Convergence. Brazilian Journalism Research, 4(2), 25-53. https://doi.org/10.25200/BJR.v4n2.2008.148

Tejedor, S. (2007). La enseñanza del ciberperiodismo. De la alfabetización digital a la alfabetización ciberperiodística. Sevilla: Comunicación Social.

Tejedor Calvo, S., \& Cervi, L. (2017). Análisis de los estudios de Periodismo y Comunicación en las principales universidades del mundo. Competencias, objetivos y asignaturas. Revista Latina de Comunicación Social, 72, 1626-1647. https://doi.org/10.4185/RLCS-2017-1238

Tejedor, S., Giraldo Luque, S., \& Carniel Bugs, R. (2018). Las redes sociales como salida professional para los estudiantes de comunicación de España y América Latina. OBS Observatorio, 12(1), 183-198. http://dx.doi.org/10.15847/ obsOBS12120181194

Toffler, A. (1980). The Third Wave. EEUU: Bantam Books.

Torres, A. (25/10/2016). La mayoría de universidades del mundo va a desaparecer. El País. Recuperado de https://goo.gl/Wc85ud (5-6-2018).

Tramullas, J. (2016). Hannibal ad portas, o los futuros perfiles profesionales de la información. El profesional de la información, 25(2),157-162. https:// doi.org/10.3145/epi.2016.mar.01

Trilling, B., \& Fadel, C. (2009). 21st Century Skills: Learning for Life in Our Times. San Francisco: Jossey-Bass.

Tuñez, M., Martínez, Y., \& Abejón, P. (2010). Nuevos entornos, nuevas demandas, nuevos periodistas. Estudios sobre el Mensaje Periodístico, 16, $79-94$. Recuperado de https://goo.gl/yJJtK3 (5-10-2018). 
United States General Accounting Office (1990). Case Study Evaluations. Washington: GAO.

Vygotsky, L. (1978). Mind in society: The development of higher psychological processes. Cambridge: Harvard University Press.

Waycott, J., Bennett, S., Kennedey, G., Dalgamo, B., \& Gray, K. (2010). Digital divides? Student and Staff perceptions of information and communication technologies. Computers \& Education, 54(4), 1202-1211. https:// doi.org/10.1016/j.compedu.2009.11.006

Fecha de recepción: 2018/11/19; Fecha de aceptación: 2019/02/08;

Fecha de publicación: 2019/03/01 